\title{
THE EFFECT OF PACKED MATERIAL ON THE NOX REMOVAL BY PACKED BEADS BARRIER DISCHARGE REACTOR
}

\author{
Student Member Toshikazu Nomura (Musashi Institute of Technology) \\ Member Yoshiyasu Ehara \\ Member Tairo Ito \\ (Musashi Institute of Technology) \\ (Musashi Institute of Technology)
}

Keywords: NOx removal, Packed beads reactor, Superimposing discharges

\section{Introduction}

Recently, air pollution has been a serious problem such as photochemical smog and acid rain are typical phenomena. NOx is one of air pollution substance and poisonous gas. So, we are longing for cutting on amount of NOx exhausted.

We have been suggested the methods of superimposing surface and silent discharges are useful on NOx removal [1-2]. We proposed a new reactor that is filled it's gap area with dielectric barrier beads since we expect that surface and silent discharge are generated simultaneously at the same space. Packed-Bed reactor like this new reactor for NOx and VOCs decomposition has been investigated by many researchers [3-6]. It has been shown that those results are good performances of NOx removal.

In this paper, the effect of packed material in the reactor such as glass, $\mathrm{Al}_{2} \mathrm{O}_{3}$ and $\mathrm{BaTiO}_{3}$ beads on the NOx removal performances is experimentally investigated.

\section{Experimental set-up}

2.1 Experimental system The sample gas was supplied from gas cylinder, $\mathrm{N}_{2}$ base containing $1650 \mathrm{ppm}$ of NO. So, the initial concentration of $\mathrm{NO}$ and $\mathrm{NOx}\left(\mathrm{NO}+\mathrm{NO}_{2}\right)$ were set at $1650 \mathrm{ppm}$ and $1680 \mathrm{ppm}$, respectively. The sample gas flow rate is from 0.4 to $1.4 \mathrm{~L} / \mathrm{min}$ For the neglect of absorption, the initial concentrations are the reading after ten-odd minutes without applied voltage. The sample gas does not contain water vapor and the others gas components for the reason that the analysis of chemical reaction in the reactor should not complicate. The concentrations of $\mathrm{NO}$ and $\mathrm{NOx}\left(\mathrm{NO}+\mathrm{NO}_{2}\right)$ in sample gas upstream and downstream of the reactor were measured by a chemiluminescence type NOx analyzer (Best Instruments: BCL511AS).

2.2 Packed beads barrier discharge reactor A schematic diagram of electrode construction of packed beads discharge rector is shown in Fig. 1. The construction of this reactor is similar to packed-bed reactor [3-6]. This reactor feature filling barrier beads as single layer and electrodes covered with glass plate. The parallel plate electrodes arrangement with an effective discharge area of $30 \mathrm{~mm} \times 300 \mathrm{~mm}$ and discharge gap of $3 \mathrm{~mm}$ filled with dielectric barrier beads ( $3 \mathrm{~mm}$ diameter) was used for discharge unit. So, surface and silent discharges may be generated simultaneously at the same space. Amplified AC $50 \mathrm{~Hz}$ high voltage from 0 to $24 \mathrm{kVpp}$ was applied to one electrode and the other electrode was grounded. Surface discharge is generated the surface of barrier bead and the point of contact beads and glass plate. Silent discharge is generated between two electrodes. The discharge current and input discharge power is determined by Lissajous figure.

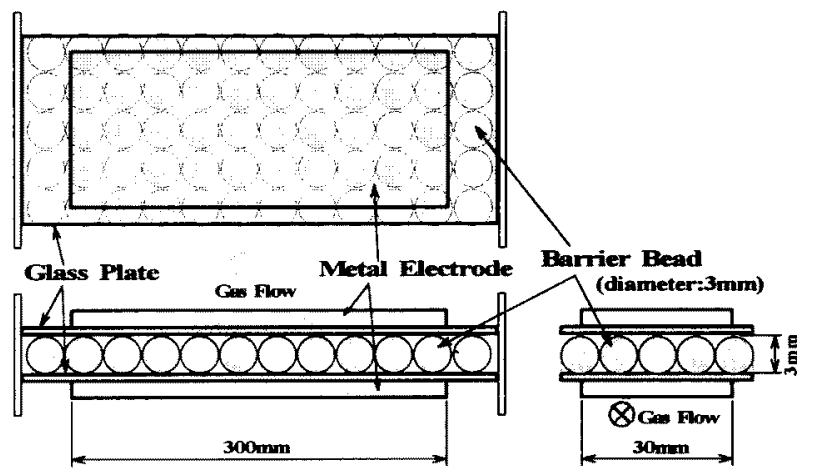

Fig. 1 Electrode construction of packed beads discharge reactor

\section{Results and discussions}

3.1 NOx removal characteristics Typical NO, NOx and $\mathrm{NO}_{2}$ concentration as a function of discharge power for the gas flow rate is $0.4 \mathrm{~L}$ min with $\mathrm{Al}_{2} \mathrm{O}_{3}$ beads condition is shown in Fig. 2. The NO and NOx concentrations decrease with increasing discharge power, on the other hand, $\mathrm{NO}_{2}$ concentration increases with increasing discharge power. However, the $\mathrm{NO}_{2}$ enhancement concentration is much lower than the NO removal concentration. This result suggests that NO removal reactions are not only oxidation reaction to $\mathrm{NO}_{2}$, but also the other byproducts such as $\mathrm{N}_{2} \mathrm{O}, \mathrm{N}_{2}$ and so on. The results on the other conditions are similar to that on the above.

3.2 The effect of barrier bead In this paragraph, the effects of barrier beads are discussed by comparison between with and without barrier beads conditions. On that time, we take note of gas flow rate and residence time. However, these elements are calculated on the assumption the atmospheric pressure without variable pressure in the reactor.

The NO removal rate as a function of gas flow rate both with and without barrier beads conditions is shown in Fig. 3. The NO 


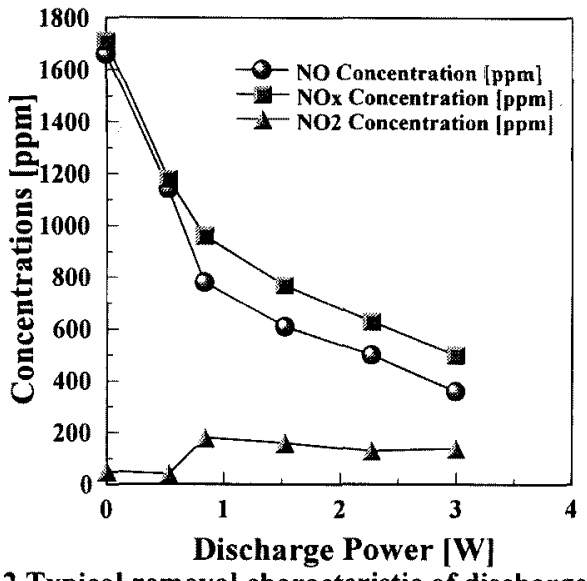

Fig. 2 Typical removal characteristic of discharge power

removal rate with barrier beads is higher than that without barrier bead. The highest removal rate of $\mathrm{NO}$ is gotten at the condition with $\mathrm{Al}_{2} \mathrm{O}_{3}$ beads. Hence, on the lower specific dielectric constant region, the higher specific dielectric constant is useful on NOx removal. However, $\mathrm{BaTiO}_{3}$ beads is shorter diameter than the others and non-similar the shape of beads. So, it is not easy to compare simply $\mathrm{BaTiO}_{3}$ beads condition with the other barrier bead conditions. Our estimation is opposite to this result since expecting few effects of variable pressure. So, pressure in the reactor rather rises at the condition with barrier bead.

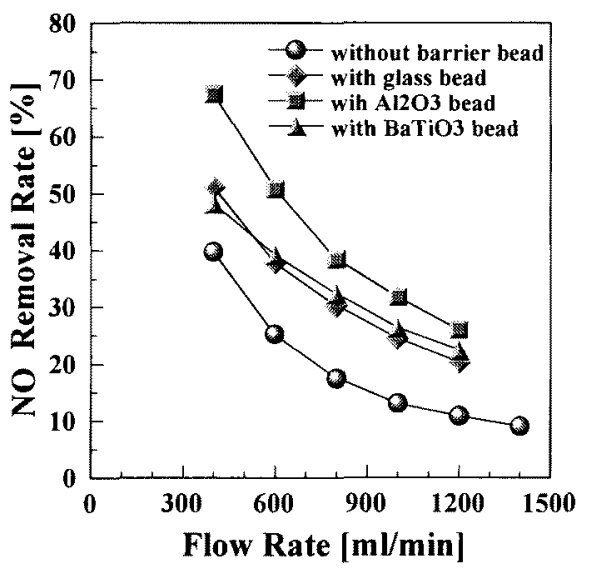

Fig. 3 NO removal rate as a function of gas flow rate

The NO removal rate as a function of gas residence time both with and without barrier beads conditions is shown in Fig. 4. The difference of removal rate at the conditions with and without barrier beads is remarkable, especially on increasing residence time. This result suggests that the discharges are generated uniformity in the gap area and NOx is removed efficiently at the condition with barrier bead. The surface and silent discharges may be generated simultaneously at the same space. On the above, at the condition with $\mathrm{Al}_{2} \mathrm{O}_{3}$ bead is most useful of removing NOx.

\section{Conclusions}

We ignore the pressure rise, we conclude that the condition with barrier beads is very effective of NOx removal. Especially, the condition with $\mathrm{Al}_{2} \mathrm{O}_{3}$ beads is useful of removing NOx. The

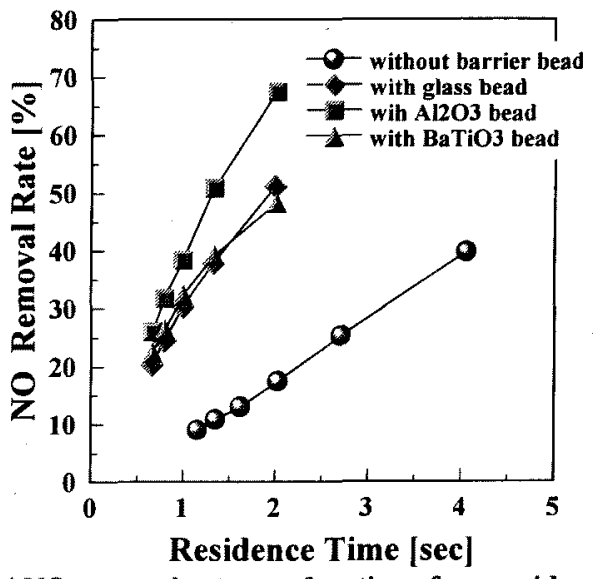

Fig. 4 NO removal rate as a function of gas residence time

higher specific dielectric constant of barrier bead is useful of removing NOx in the lower specific dielectric constant region.

In this paper, the sample gas does not contain water vapor, soot and so on, but real exhaust gases contains. We will investigate the effect of water vapor using simulated exhaust gas. On the other hand, concurrent use of DPF (Diesel Particulate Filter) will be serving a useful function for soot.

Authors wish to thank YUGYOKUEN Co.,Ltd., Fukushima Ceramic Co.,Ltd. and Prof. J.S. Chang at McMaster Univ. for supplying beads to us.

(Manuscript received Dec. 16, '97, revised Feb. 15, '98)

\section{References}

[1] H. Miyamoto, et.al., "The Effect of Superimposed Discharge on the Decomposition of NOx in a Exhaust Gas", T. IEE Japan, 116-A, No.8, pp.678-683(1996)

[2] T. Nomura, et.al., "The Effect of Discharge Condition on NOx Reduction by Superimposing Surface and Silent Discharges", T. IEE Japan, 118-A, No.4, pp.341-346(1998)

[3] H.H. Kim, et.al., "A Novel Plasma Reactor for NOx Control Using Photocatalyst and Hydrogen Peroxide Injection", Proc. IEEE IAS 32nd Annual Meeting, pp.1937-1941(1997)

[4] T. Yamamoto, et.al., "Plasma-assisted Chemical Reactor for NOx Decomposition", Proc. IEEE IAS 32nd Annual Meeting, pp.1956-1960(1997)

[5] A. Ogata, et.al., "Decomposition of Benzene Using NonThermal Plasma Reactor Packed with Ferroelectric Pellet", Proc. IEEE IAS 32nd Annual Meeting, pp.1975-1982(1997)

[6] T. Kawasaki, et.al., "The Effect of Operational Time of $\mathrm{BaTiO}_{3}$ Pellets on NOx Removal in a Packed-bed Plasma Reactor", Proc. 1998 Annual Meeting of The Institute of Electrostatics Japan, pp.205-206(1998)

Toshikazu Nomura (Student Member) Refer to Trans. of IEE Japan, 118-A, No.4, p.346 (1998)

Yoshiyasu Ehara (Member) Refer to Trans. of IEE Japan, 119-A, No.3, p.272 (1999)

Tairo Ito (Member) Refer to Trans. of IEE Japan, 119-A, No.3, p.272 (1999) 